\title{
Preparation and Characterization of Anti-GP73 Monoclonal Antibodies and Development of Double-antibody Sandwich ELISA
}

\author{
Qi-Wen Li ${ }^{1 \&}$, Hong-Bing Chen ${ }^{2 \&}$, Zhi-Yang Li ${ }^{1}$, Peng Shen ${ }^{3}$, Li-Li Qu ${ }^{1}$, Lai-Ling \\ Gong ${ }^{1}$, Hong-Pan $\mathrm{Xu}^{1}$, Lu Pang ${ }^{1}$, Jin $\mathrm{Si}^{1 *}$
}

\begin{abstract}
Background: Serum Golgi protein 73 (GP73) as a novel and potential marker for diagnosing hepatocellular carcinoma (HCC) have been found to be elevated in HCC patients and associated with clinical variables representing tumor growth and invasiveness. The aim of this study was to prepare a pair of monoclonal antibodys (mAbs) against GP73 and develop a newly designed double-antibody sandwich enzyme-linked immunosorbent assay (s-ELISA), which would be used in the detection of serum GP73 (sGP73) as well as in the diagnosis of HCC. Materials and Methods: Produced by prokaryotic expression, the purified recombinant GP73 (rGP73), produced by prokaryotic expression, was used to immunize the Balb/c mice. Two hybridoma cell lines against GP73 were obtained by fusing mouse $\mathrm{Sp2} / 0$ myeloma cells with spleen cells from the immunized mice. The titers of anti-GP73 mAb reached 1:243,000. Western blotting analysis and Immunohistochemistry staining revealed that anti-GP73 mAb could recognize GP73 protein. The double-antibody s-ELISA was successfully established and validated by $119 \mathrm{HCC}$ and 103 normal serum samples. Results: showed that the detection limit of this method could reach $1.56 \mathrm{ng} / \mathrm{ml}$, and sGP73 levels in $\mathrm{HCC}$ group (mean=190.6 ng/ml) were much higher than those of in healthy controls (mean=70.92 ng/ml). Conclusions: Results of our study not only showed that sGP73 levels of HCC patients were significantly higher than those of healthy controls, but also indicated that the laboratory homemade anti-GP73 mAbs could be the optimal tool used in evaluating sGP73 levels, which would provide a solid foundation for subsequent clinical applications.
\end{abstract}

Keywords: GP73 - HCC - monoclonal antibody - serum marker - ELISA

Asian Pac J Cancer Prev, 16 (5), 2043-2049

\section{Introduction}

Hepatocellular carcinoma (HCC) ranks among one of the most common primary malignant cancers worldwide. A great number of chronically infected people, such as hepatitis $\mathrm{B}$ virus (HBV) or hepatitis $\mathrm{C}$ virus (HCV) infected individuals (Al-Kubaisy et al., 2014; Li et al., 2014; Munaf et al., 2014; Somboon et al., 2014), gradually become severe liver cirrhosis and then may finally progress to HCC. HCC is the fifth most common cancer in men $(523,000$ cases, $7.9 \%$ of the total $)$, the seventh in women $(226,000$ cases, $6.5 \%$ of the total), and the third leading cause of cancer-related mortality (Ferlay et al., 2010). Although prevalence remains highest in Eastern Asia and Africa (Thun et al., 2010), the incidence rates of HCC steadily increased in the Western world over the last 30 - 50 years (Sherman, 2010) mainly due to the late clinical diagnosis and therapy of most patients. Therefore, in order to provide patients with higher survival rate, early detection and surgical extraction are in urgent demand (Stefaniuk et al., 2010; Masuzaki et al., 2012). At present, CT, MRI and biomarker could help establish noninvasive diagnosis, and the use of ultrasonography with or without serum alpha-fetoprotein (AFP) determination was recommended for screening and surveillance (Sangmala et al., 2014), Serum AFP is the most commonly used biomarker for HCC, but the clinical diagnostic accuracy to detect early $\mathrm{HCC}$ has been questioned as its sensitivity and specificity are only around 60\% (Marrero et al., 2009; Lok et al., 2010). A large number of HCC patients have AFP levels below the critical value which is difficult to undergo detection and prognosis of HCC (Yamashita et al., 2008).

GP73, a novel Golgi-localized protein initially reported by Kladney RD (Kladney et al., 2000), is normally expressed in biliary epithelial cells of human tissues. It is overexpressed in patients with acute and chronic hepatitis and liver cirrhosis (Liu et al., 2011), and

${ }^{1}$ Department of Laboratory Medicine, The Second Affiliated Hospital, Nanjing Medical University, ${ }^{3}$ Institute of Digestive Endoscopy and Medical Center for Digestive Diseases, The Second Affiliated Hospital, Nanjing Medical University, ${ }^{2}$ Department of Laboratory Medicine, Nanjing Children's Hospital, Nanjing Medical University, Nanjing, China \&Equal contributors *For correspondence: sijin_efy@njmu.edu.cn 
the levels in the HCC group are much higher than in the healthy and other liver disease group (Fimmel and Wright, 2009; Shan et al., 2013; Qiao et al., 2014). Recently, accumulating studies revealed that the sensitivity and specificity of GP73 for HCC diagnosis were more superior than AFP (Hu et al., 2010; Zhou et al., 2012; Hou et al., 2013; Hu et al., 2013), and a meta-analysis (Witjes et al., 2013) also indicated that GP73 was superior to AFP for the early diagnosis and screening of HCC, so GP73 was proposed as a novel surrogate marker for HCC diagnostics. In addition, GP73 levels were reported to be associated with clinical variables representing tumor growth and invasiveness (Riener et al., 2009; Hu et al., 2010; Wang et al., 2014). Therefore, it is very meaningful to measure the expression level of GP73.

Immunoblotting, the gold standard of protein analysis, has been used in various studies at present, but it has inherent limitations (e.g. semi-quantitative and laborious) and suffers from widespread applications. However, the enzyme-linked immunosorbent assay (ELISA), which is more efficient and convenient, is a preferable methodology in sGP73 quantitation analysis. At present, many research groups have obtained a consistent conclusion that sGP73 was expressed significantly higher in HCC subjects than in healthy controls, but the sGP73 concentration reported by those groups was dissimilar, which was most likely due to the methodological bias and the specificity of ELISA (Zhang and Cao, 2012). Therefore, an anti-GP73 antibody with high specificity and affinity was demanded.

As monoclonal antibodies possess high specificity, we established hybridoma cell lines that stably secreted anti-GP73 mAb in the study. After been purified and evaluated by Western blotting and immunohistochemistry, a pair of anti-GP73 mAbs was used for developing a double-antibody s-ELISA. This newly designed method was further validated by normal serum and HCC serum samples. The obtained results not only demonstrated that the expression levels of sGP73 were substantially increased in HCC patients compared with healthy controls, but also revealed that anti-GP73 mAb produced by hybridoma technique laid a foundation for subsequent clinical applications and provided a basis for diagnostic reagent preparation.

\section{Materials and Methods}

\section{Materials and clinical serum samples}

Plasmid pCold II, BL21 (DE3) host strain E.coli, mouse Sp2/0 myeloma cells, human hepatoma cell line SMMC-7721, human hepatoma cell line HepG2, and normal human hepatocyte line LO2 were laboratory stored. Freund's complete adjuvant, Freund's incomplete adjuvant, horseradish peroxidase (HRP) conjugated goat anti-mouse IgG, Bovine Serum Albumin (BSA), and Mouse Monoclonal Antibody Isotyping Reagents were purchased from Sigma Chemical Co. (St. Louis, MO, USA). Polyethylene glycol 1500 (PEG 1500) was purchased from Roche Diagnostics (Mannheim, Germany). Dulbecco's Modified Eagle Medium (DMEM) was purchased from Hyclone Laboratories (Logan, UT). Restriction endonuclease (Nde I and Hind III) was purchased from Invitrogen (USA). Flat-bottomed polystyrene 96-well micro-titer plates were purchased from Corning (USA). ELISA reader (Bio-Rad ELISA Reader) was obtained from Japan. BCA Protein Assay Kit was purchased from KeyGEN Biotech (Nanjing, China).

Female Balb/c mice (6-8 weeks) were obtained from experimental animal center of our university. A total of 222 serum samples were obtained from the Second Affiliated Hospital of Nanjing Medical University (Nanjing, China), including 119 HCC (median age, 58 years old; 72 males and 47 females), and 103 healthy (median age, 52 years old; 61 males and 42 females) individuals. The human reference serum was analyzed without any pretreatment.

\section{Expression and purification of rGP73}

The restriction fragment of GP73-short cDNA from HepG2 cells was initially amplified by polymerase chain reaction (PCR) using a pair of primers: 5'-AAGCTTTCAGAGTGTATGATTCCGC-3' and 5'-CATATGAGCTCCCGGAGCGTGGACC-3', and then the resultant amplified products were inserted into the pCold II vector with a N-terminal $6 \times$ histidine tag containing Nde I and Hind III restriction sites. Subsequently, confirmed by restriction enzyme digestions and sequence analysis, the recombinant plasmid pCold II-GP73-short was transformed into E.coli BL21 (DE3) with standard procedures to induce E.coli BL21 (DE3) to express rGP73 with $0.5 \mathrm{mM}$ isopropy- $\beta$-D-thiogalactoside (IPTG) for 24 hours at $16^{\circ} \mathrm{C}$. After that, bacteria were resuspended in $20 \mathrm{~mL}$ lysis buffer $(20 \mathrm{mM}$ Tris- $\mathrm{HCl}$ containing $1 \mathrm{mM}$ PMSF and bacteria protease inhibitor cocktail, $\mathrm{pH}$ 8.0) and sonicated on ice until they were lysed, which were followed by the sodium dodecyl sulphate-polyacrylamide gel electrophoresis (SDS-PAGE) to monitor the expression and the expression form.

According to the manufacturer's instructions, the obtained GP73 fusion proteins (namely rGP73) were purified with His Trap HP affinity column (GE Healthcare) and desalted with HiTrap Desalting column (GE Healthcare). Finally, the target proteins were eluted with $1 \times \mathrm{PBS}(\mathrm{pH} 7.4)$ during desalting and stored at $-80^{\circ} \mathrm{C}$ after been validated by SDS-PAGE and Western blotting.

\section{Animal immunization and generation of hybridomas}

The methods of animal immunization and hybridoma production were similar to what were described by Kaushal (2014) and Xu (2014). Briefly, mouse anti-GP73 antibodies were produced by intraperitoneally injecting female BALB/c mice (6-8 weeks) with purified rGP73 (100 $\mu \mathrm{g} / \mathrm{mouse})$ suspended in Freund's complete adjuvant, followed by additional boost injections in Freund's incomplete adjuvant at 2 -week intervals. The mouse showing the highest antibody titer was given the final boost intravenous injection 3 days before fusion. Splenocytes of the sacrificed mice were fused with $\mathrm{Sp} 2 / 0$ myeloma cells according to the standard techniques. Hybridoma clones were screened by hypoxanthine-aminoprerin-Thymidine (HAT) medium and the supernatant of culture medium was tested by indirect ELISA. The hybridoma clones with consistent reactivity with rGP73 were selected and expanded in in vitro cultures. Finally, $0.5 \mathrm{~mL}$ of hybridoma 
cells $\left(2 \times 10^{6} / \mathrm{ml}\right)$ were intraperitoneally injected into pristane primed mice for mAbs production in ascites.

\section{Isotyping and purification of $m A b s$}

Isotyping of mAbs were determined by mouse monoclonal antibody isotyping reagents. The mAbs (designated as 8E7, 5A10, respectively) were purified with a protein-A column (GE Healthcare) and then desalted with HiTrap Desalting column (GE Healthcare) according to the manufacturer's instructions. The purity of mAbs was analyzed by SDS-PAGE, and the concentration of mAbs was determined by BCA Protein Assay Kit.

\section{ELISA}

The titers of purified mAbs obtained from mice ascites were detected by indirect ELISA. ELISA plates were precoated with purified rGP73 $(5 \mu \mathrm{g} / \mathrm{ml})$ and blocked with Tris-buffered saline (TBS)-Tween (TBST, containing $1 \times \mathrm{TBS}$ and $0.05 \%$ Tween 20$)$ containing $1 \%(\mathrm{w} / \mathrm{v})$ bovine serum albumin (BSA). Afterwards, the purified mAbs (8E7 or $5 \mathrm{~A} 10)$ were added into the plates with serial dilutions ( $1.56 \mathrm{ng} / \mathrm{ml}$ was the initial concentration), followed by the incubation with horseradish peroxidase (HRP) conjugated goat anti-mouse IgG. After washing four times, TMB substrate solution was added, followed by the addition of $\mathrm{H}_{2} \mathrm{SO}_{4}(2 \mathrm{M})$ to stop the reaction. Absorbance of 450 $\mathrm{nm}(\mathrm{A} 450 \mathrm{~nm})$ was measured to determine the titers of purified mAbs.

\section{Western blotting}

Western blotting enables the detection of interest proteins using primary antibodies. Employing discontinuous polyacrylamide gel electrophoresis and nitrocellulose membrane, it can separate proteins according to their molecular weights. To validate the specific reaction of the purified $m A b s$ to $\mathrm{GP} 73$ and natural GP73 (nGP73), the rGP73 as well as nGP73 extracted respectively from HepG2 cells, SMMC-7721 cells and LO2 cells were subjected to western blotting assays. In this experiment, membranes were blocked with 5\% nonfat milk in TBST for $1 \mathrm{~h}$ at room temperature and then incubated with 8E7 or 5A10 (1:1000 dilution in 5\% nonfat milk/TBST) overnight at $4^{\circ} \mathrm{C}$, followed by the addition of HRP conjugated goat anti-mouse IgG as secondary antibody (1:5000 dilution in TBST) to incubate the membranes for $1 \mathrm{~h}$ at room temperature. After a final wash with TBST in triplicate, the membranes were detected with enhanced chemiluminescence system.

\section{Immunohistochemistry staining}

According to the suggested immunohistochemistry protocol, tissue section slides went through deparaffinization by xylene, rehydration by graded alcohol and antigen retrieval. After applying 5\% BSA to block the slides, diluted anti-GP73 mAb (8E7 or 5A10, 1:400) was added and incubated overnight at $4^{\circ} \mathrm{C}$. The location of primary antibody ( $8 \mathrm{E} 7$ or $5 \mathrm{~A} 10$ ) was determined by a biotinylated anti-primary antibody, which could conjugate to HRP and diaminobenzidine through biotin-streptavidin interactions (Dako, Glostrup, Denmark). After that, the slides were counterstained by hematoxylin.

\section{Double-antibody s-ELISA}

According to the manufacturer's instructions, 8E7 and 5A10 were respectively conjugated to HRP using Activated Horseradish Peroxidase Labeling Kit (GalaxyBio, China). To investigate the optimal experimental conditions for double-antibody s-ELISA, 8E7 and 5A10 as coating antibody were respectively added into micro-titer plates with a series of concentrations $(5,10,15,20 \mu \mathrm{g} / \mathrm{ml})$ and incubated overnight at $4^{\circ} \mathrm{C}$. Blocked by $1 \% \mathrm{BSA} / \mathrm{TBST}$ at $37^{\circ} \mathrm{C}$ for $2 \mathrm{~h}$, the micro-titer plates were incubated with two-fold serial dilutions of purified rGP73 at $37^{\circ} \mathrm{C}$ for 1 h. After washed with TBST for three times, the microtiter plates coated with 8E7 and 5A10 were respectively incubated with HRP-5A10 and HRP-8E7 with different dilutions $(1: 500,1: 1000,1: 2000)$ for $1 \mathrm{~h}$ at $37^{\circ} \mathrm{C}$, followed by the addition of TMB substrate solution. The absorbance was measured at $450 \mathrm{~nm}$ when the reaction stopped. In this experimentation, rGP73 (200 ng/ml) and 1\% BSA/TBST acted as positive control and negative control respectively, and the relationship between $\mathrm{A}_{450 \mathrm{~nm}}$ and GP73 antigen concentration was analyzed applying CurveExpert1.3.

To assess the performance of double-antibody s-ELISA in clinical serum samples, the expression levels of sGP73 in patients with HCC $(n=119)$ and healthy controls $(n=103)$ were analyzed. Each well of the micro-titer plate was coated with $100 \mu \mathrm{l} 8 \mathrm{E} 7(5 \mu \mathrm{g} / \mathrm{ml})$ prior to overnight incubation at $4^{\circ} \mathrm{C}$. After washed with TBST, each well was blocked by blocking solution (TBST containing 1\% BSA) at $37^{\circ} \mathrm{C}$ for $2 \mathrm{~h}$, and ten-fold diluted sera from healthy and $\mathrm{HCC}$ subjects were added respectively into the micro-titer plates $(100 \mu \mathrm{l} /$ well $)$ for incubation at $37^{\circ} \mathrm{C}$ for $1 \mathrm{~h}$. The plates were washed for 3 times, followed by the addition of HRP-5A10 (dilution 1:500 in 1\% BSA/TBST) for $1 \mathrm{~h}$ at $37^{\circ} \mathrm{C}$. At last, TMB substrate solution was added and the measurement of $\mathrm{A}_{450 \mathrm{~nm}}$ was carried out.

\section{Statistical analysis}

Statistical analysis was performed using GraphPad Prism 5.0. Differentiation of sGP73 concentrations between two groups (healthy controls and HCC group) were compared according to a two-tailed Student's t-test. P-values $<0.05$ were considered to be statistically significant.

\section{Results}

\section{Expression and purification of recombinant protein}

The prokaryotic expressed recombinant plasmid pCold II-GP73-short was successfully constructed and verified by complete sequencing and restriction enzyme digestions (Figure 1A). According to SDS-PAGE analysis of GP73 expression, the recombinant protein was successfully expressed and existed in the supernatant of centrifuged bacterial lysate after induced (Figure 1B). After the bacterial lysate was centrifuged, the supernatant was collected and subsequently loaded onto the affinity column to get purified target protein. The purity of the target protein analyzed by SDS-PAGE was $>90 \%$. The molecular weight of the recombinant GP73 protein was $55 \mathrm{kDa}$ (Figure 1C), which was perfectly consistent with prediction. 


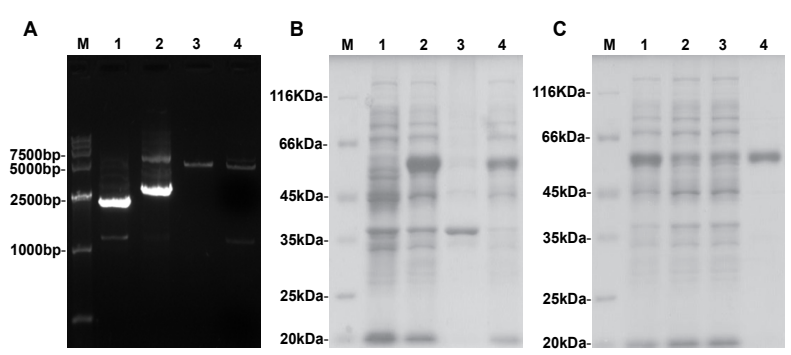

Figure 1. Restriction Digestion of pCold II and pCold II-GP73-short Recombinant Plasmids, and SDS-PAGE Analysis of Purified Recombinant GP73. A) Lane M: DNA Marker D15000, Lane 1: empty plasmid pCold II without digestion, Lane 2: pCold II-GP73-short without digestion, Lane 3: empty plasmid pCold II after digestion, Lane 4: recombinant plasmid pCold II-GP73-short after digestion. It showed that prokaryotic expressed recombinant plasmid pCold II-GP73-short was successfully constructed. B) Lane M: protein marker, Lane 1: inoculated LB before induced, Lane 2: inoculated LB after induced at $16^{\circ} \mathrm{C}$ for $24 \mathrm{~h}$, Lane 3: the precipitate of centrifuged bacterial lysate, Lane 4: the supernatant of centrifuged bacterial lysate. It showed that GP73 fusion protein was successfully expressed and existed in the supernatant of centrifuged bacterial lysate. C) Lane M: protein marker, Lane 1: the supernatant of centrifuged bacterial lysate after induced, Lane 2: flow-through, Lane 3: washed with wash buffer, Lane 4: the purified GP73 fusion protein eluted from the column. Proteins were visualized by Coomassie Blue R-250 staining

\section{Generation of hybridoma cell lines and purification of $m A b s$}

As the healthy female Balb/c mice immunized with purified rGP73 could generate antibody against GP73, the blood samples of these mice were investigated to determine the antiserum titer by indirect ELISA. In this experiment, purified rGP73 $(5 \mu \mathrm{g} / \mathrm{ml})$, which acted as coating antigen, was added into the micro-titer plates (100 $\mathrm{ml} /$ well). The results indicated that rGP73 as immune antigen successfully induced generation of antibody against GP73 and the antibody titers in mice reached up to $1: 121,500$. Then the spleen cells of mice with the highest titer were fused with $\mathrm{Sp} 2 / 0$ myeloma cells and two hybridoma cell lines against GP73 were successfully obtained. Anti-GP73 mAbs stably produced by those two hybridoma cell lines were designated as $8 \mathrm{E} 7,5 \mathrm{~A} 10$, respectively.

These two hybridoma cell lines were injected respectively into the abdominal cavity of female Balb/c mice, and a large quantity of mice ascites containing $8 \mathrm{E} 7$ or $5 \mathrm{~A} 10$ were generated. The SDS-PAGE analysis demonstrated that the two affinity-purified anti-GP73 $\mathrm{mAbs}$ had a band of heavy chain at $50 \mathrm{kDa}$ and a band of light chain at $25 \mathrm{kDa}$, and the purity reached $>90 \%$.

\section{Characteristics of anti-GP73 $m A b s$}

Characterization of anti-GP73 mAbs was carried out to determine their clinical application value and make full use of them in disease diagnostics. The titers of mAbs were tested by indirect ELISA, which indicated that all of the mAbs reached up to 1: 243,000 , and the actual concentrations of 8E7 and 5A10 at dilution of 1:243,000 were $2.47 \mathrm{ng} / \mathrm{ml}$ and $3.29 \mathrm{ng} / \mathrm{ml}$ respectively (Figure $2 \mathrm{~A})$. The subtypes of $8 \mathrm{E} 7$ and $5 \mathrm{~A} 10$ were determined by

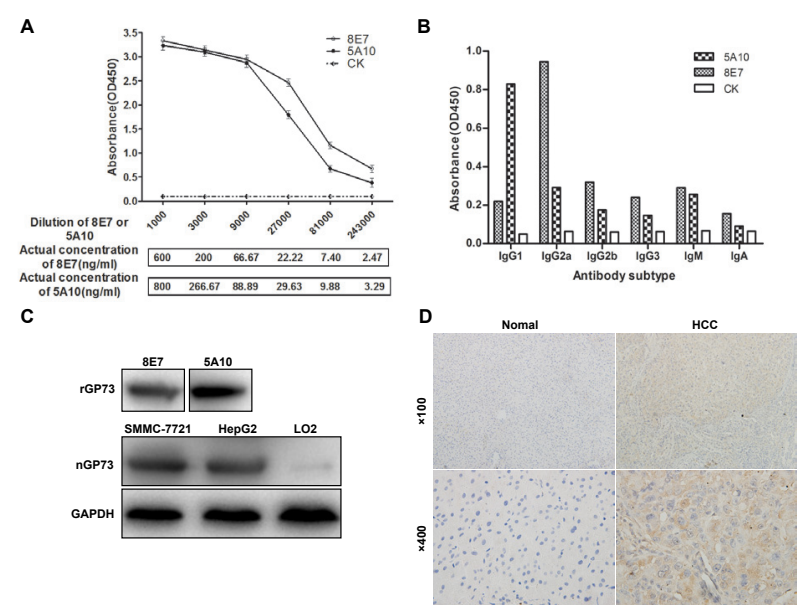

Figure 2. Characteristics of anti-GP73 mAbs. A) Titer results of 8E7, 5A10 by indirect ELISA showed that all of two $\mathrm{mAbs}$ had high titers $(>1: 243,000)$ and the actual concentrations of $8 \mathrm{E} 7$ and 5A10 at dilution of 1:243,000 were $2.47 \mathrm{ng} / \mathrm{ml}$ and $3.29 \mathrm{ng} / \mathrm{ml}$, respectively. The measurements at each dilution were taken in triplicate. CK: $1 \%$ BSA/TBST. B) The subtypes of anti-GP73 mAbs were determinated by Isotyping (IgG1, IgG2a, IgG2b, IgG3, IgM, IgA) Kit, and the subtypes of 8E7, $5 \mathrm{~A} 10$ were IgG2a, IgG1, respectively. CK: $1 \times$ PBS. C) The specificity of $\mathrm{mAbs}$ was tested by western blotting assays with recombinant GP73 (rGP73) and native GP73 (nGP73). The rGP73 protein produced by prokaryotic expression was recognized by $8 \mathrm{E} 7$ as well as 5A10 perfectly. To investigate whether our mAb could recognize nGP73, cell lysates of SMMC-7721, HepG2 and LO2 were respectively subjected to western blotting analysis using 8E7. GAPDH was used as an internal control. The results showed the specific reaction of $8 \mathrm{E} 7$ to $\mathrm{nGP73}$ and the higher expression level of GP73 in hepatoma cells than in normal hepatocyte cells (5A10 brought the same result in western blotting assays with nGP73). D) Immunohistochemistry staining using anti-GP73 $\mathrm{mAb}$ on sections of HCC and normal liver tissues. GP73 staining mainly focused on the perinuclear region of HCC cells, which showed the specific reaction of mAb to GP73 and preliminary demonstrated that the concentration of GP73 in HCC patients was higher than that in healthy controls. The mAb uesd in this figure was $8 \mathrm{E} 7$, and $5 \mathrm{~A} 10$ brought the same results in immunohistochemistry staining

Isotyping ( $\operatorname{IgG} 1$, IgG2a, $\operatorname{IgG} 2$ b, $\operatorname{IgG} 3, \operatorname{Ig} M, \operatorname{Ig} \mathrm{A}) \mathrm{Kit}$, and the results showed that the isotypes of $8 \mathrm{E} 7$ and $5 \mathrm{~A} 10$ were IgG2a, IgG1, respectively (Figure 2B). The specificity of mAbs was tested by western blotting assays with rGP73 and native GP73 (nGP73). As shown in Figure 2C, the rGP73 protein produced by prokaryotic expression was recognized by $8 \mathrm{E} 7$ and $5 \mathrm{~A} 10$. To investigate whether $8 \mathrm{E} 7$ or $5 \mathrm{~A} 10$ could recognize nGP73 or not, we extracted the nGP73 protein from SMMC-7721, HepG2 and LO2 cells, all of which expressed GP73 positively. The details in Figure $2 \mathrm{C}$ showed the specific reaction of mAbs with nGP73 and the increasing expression level of GP73 in hepatoma cells. In addition, immunohistochemistry analysis was also performed to validate the specificity of mAbs to GP73 protein. As shown in Figure 2D, the specificity of our mAbs was satisfactory. Those analysis results revealed that anti-GP73 mAbs with high titers and high specificity were successfully obtained.

\section{Standard curves}

In the double-antibody s-ELISA, the utilization of 

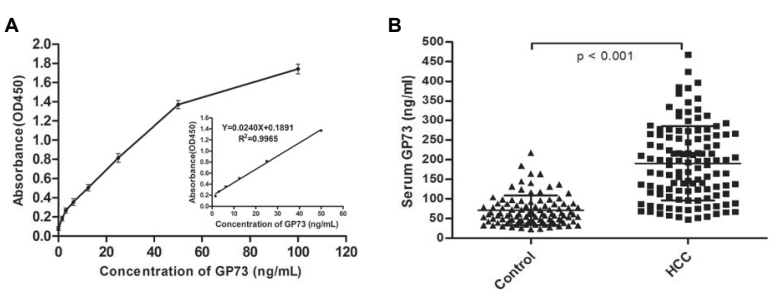

Figure 3. Standard Curve and Detection of sGP73 Levels by Double-antibody s-ELISA. A) In the doubleantibody s-ELISA, the standard curve was established with rGP73 concentration of $0,1.56,3.125,6.25,12.5,25,50$ and $100 \mathrm{ng} / \mathrm{ml}$. The inset figure showed a very good linear response in the concentration range from 1.56 to $50 \mathrm{ng} / \mathrm{ml}$ with a linear equation of $y=0.0240 x+0.1891$ and a correlation coefficient $\left(\mathrm{R}^{2}\right)$ of 0.9965 . The measurements at each concentration were carried out in quadruplicate. B) The statistical difference between 119 HCC patients and 103 healthy individuals was significant. Results were expressed as mean of triplicate experiments

coating antibody $8 \mathrm{E} 7(5 \mu \mathrm{g} / \mathrm{ml})$ and HRP conjugated antibody 5A10 (1:500 diluted) brought about higher absorbance and lower negative background. On the basis of optimal experimental conditions, the rGP73 protein was added with serial dilutions $(1.56 \mathrm{ng} / \mathrm{ml}$ as the initial concentration), and the measurements at each dilution were taken in quadruplicate at different times. The relationship between $\mathrm{A}_{450 \mathrm{~nm}}$ and GP73 antigen concentration was obtained, which showed a very good linear response in the concentration range from 1.56 to 50 $\mathrm{ng} / \mathrm{ml}$. The standard curve of double-antibody s-ELISA was established according to the following formula: $\mathrm{y}=0.0240 \mathrm{x}+0.1891, \mathrm{R}^{2}=0.9965$ (Figure 3A).

Validation of double-antibody s-ELISA in clinical samples

Clinical sGP73 samples in 103 healthy individuals and 119 HCC patients were used to validate the established double-antibody s-ELISA. The results suggested that double-antibody s-ELISA assay have been successfully developed for the quantification of sGP73, and the expression concentration of sGP73 in healthy individuals ranged from 22.8 to $217.3 \mathrm{ng} / \mathrm{ml}$, with a median of 61.6 $\mathrm{ng} / \mathrm{ml}$ and a mean of $70.92 \mathrm{ng} / \mathrm{ml}$ (95\% IC: $63.6-78.25$ $\mathrm{ng} / \mathrm{ml}$ ), while the sGP73 in HCC patients ranged from 47.8 to $467.5 \mathrm{ng} / \mathrm{ml}$, with a median of $187.3 \mathrm{ng} / \mathrm{ml}$ and a mean of $190.6 \mathrm{ng} / \mathrm{ml}$ (95\% IC: $173.5-207.7 \mathrm{ng} / \mathrm{ml})$. The statistical difference between HCC patients and healthy individuals was significant ( $<0.001$, Figure 3B).

\section{Discussion}

GP73 is a resident type II Golgi transmembrane protein expressed primarily in epithelial cells of human tissues (Kladney et al., 2000). Its expression was markedly upregulated in hepatocytes from patients with viral and non-viral liver diseases, especially from HCC patients (Hu et al., 2010; Qiao et al., 2014). Recently, many studies have documented the potential role of GP73 in HCC (Wang et al., 2009; Yamamoto et al., 2010; Sun et al., 2011; Bao et al., 2013). GP73 as an accurate serum biomarker has high sensitivity and specificity for HCC diagnosis (Hu et al., 2010; Mao et al., 2010; Zhou et al., 2012), and it is possible to be utilized in the detection of the early stage of progression of HCC (Block et al., 2008; Ozkan et al., 2011; Hu et al., 2013). GP73 levels were observed to be elevated in serum of $72.5 \%$ of HCC patients who had AFP levels less than $400 \mathrm{ng} / \mathrm{ml}$ (Zhao et al., 2010), and the combination of GP73 and AFP could achieve an ultrasensitive detection compared with either GP73 or AFP alone, which indicated an appealing clinical strategy with high sensitivity and specificity for HCC diagnosis (Hou et al., 2013; Wang et al., 2014). In particular, it was observed that the expression of GP73 was significantly associated with tumor grade (Riener et al., 2009; Hu et al., 2010) and invasiveness such as tumor number, the presence of vascular invasion, advanced stage, deteriorated liver function, and poor performance status (Wang et al., 2014), which indicated that GP73 would also be a potential predictor for HCC prognosis. Therefore, it is meaningful to measure the expression level of GP73, and the development of an ultrasensitive quantitative immunological detection reagent not only possesses scientific importance, but also makes a contribution to the HCC related field.

In our study, rGP73 protein was expressed in the E.coli heterologous protein expression system. Two hybridoma cell lines against GP73 were produced by B cell hybridoma technique and characterized by ELISA, Western blotting and immunohistochemical analysis. These assay results validated the high specificity of our mAbs against GP73. Afterwards, we successfully established the doubleantibody s-ELISA using antibody pairs (8E7 as coating antibody and 5A10 as HRP conjugated antibody). Four independent standard curves were constructed at different times, demonstrating that the system is reliable and reproducible. The detection limit of this system was as low as $1.56 \mathrm{ng} / \mathrm{ml}$, indicating the high sensitivity. In addition, the concentrations of sGP73 in HCC patients and healthy controls were measured and the results suggested that the sGP73 levels in HCC patients were significantly higher than in healthy controls $(\mathrm{p}<0.001)$, which was consistent with previous reports. At present, the expression amounts of sGP73 has been reported by several research groups, but there was a discrepancy in quantification results. The concentration of sGP73 reported by Gu et al. (2009) was higher than what was reported by Zhang and Cao (2012), but was lower than the data measured in our study. This conflict probably attributed to the specificity and affinity of GP73 antibodies, which were important factors for ELISA sensitivity. Additionally, the majority of antibodies used in previous studies of GP73, such as the study performed by $\mathrm{Gu}$ and Zhang, was polyclonal antibody. The polyclonal antibody cannot be satisfied for the specificity and affinity to GP73, and the polyclonal antibody originating from different production batches is hard to be standardized. However, the mAbs developed in this study were highly specific to the N-terminus of the GP73 fragment, and the antibody pairs used in s-ELISA could facilitate to recognize GP73 with secure affinity.

In conclusion, the anti-GP73 mAbs were successfully prepared for the double-antibody s-ELISA, the results of our study not only showed that the mAbs against GP73 laid a foundation for subsequent clinical applications and promoted the preparation of follow-up testing reagent, 
but also indicated that GP73 as a serum biomarker had a promising future in the diagnosis of HCC.

\section{Acknowledgements}

We acknowledge the financial support of the National Natural Science Foundation of China (No. 81472831, 61201033), the Medical Key Talent Foundation of Jiangsu Province (RC2011081), the Medical Key Science and Technology Development Projects of Nanjing (ZKX11176), the Talents Planning of Six Summit Fields of Jiangsu Province (2013-WSN-054, 2013-WSN056), China Postdoctoral Science Foundation funded project (2014M551491), Jiangsu Planned Projects for Postdoctoral Research Funds (1302007A), and the Science and Technology Development Fund of Nanjing Medical University (2014NJMU138).

\section{References}

Al-Kubaisy WA, Obaid KJ, Noor NA, et al (2014). Hepatitis $\mathrm{C}$ virus prevalence and genotyping among hepatocellular carcinoma patients in Baghdad. Asian Pac J Cancer Prev, 15, 7725-30.

Block TM, Marrero J, Gish RG, et al (2008). The degree of readiness of selected biomarkers for the early detection of hepatocellular carcinoma: Notes from a recent workshop. Cancer Biomark, 4, 19-33.

Bao YX, Cao Q, Yang Y, et al (2013). Expression and prognostic significance of golgiglycoprotein73 (GP73) with epithelialmesenchymal transition (EMT) related molecules in hepatocellular carcinoma (HCC). Diagn Pathol, 8, 197.

Fimmel CJ, Wright L (2009). Golgi protein 73 as a biomarker of hepatocellular cancer: development of a quantitative serum assay and expression studies in hepatic and extrahepatic malignancies. Hepatology, 49, 1421-3.

Ferlay J, Shin HR, Bray F, et al (2010). Estimates of worldwide burden of cancer in 2008: GLOBOCAN 2008. Int J Cancer, 127, 2893-917.

Gu Y, Chen W, Zhao Y, et al (2009). Quantitative analysis of elevated serum golgi protein-73 expression in patients with liver diseases. Ann Clin Biochem, 46, 38-43.

Hu JS, Wu DW, Liang S, Miao XY (2010). GP73, a resident golgi glycoprotein, is sensibility and specificity for hepatocellular carcinoma of diagnosis in a hepatitis B-endemic Asian population. Med Oncol, 27, 339-45.

Hu B, Tian X, Sun J, et al (2013). Evaluation of individual and combined applications of serum biomarkers for diagnosis of hepatocellular carcinoma: a meta-analysis. Int J Mol Sci, 14, 23559-80.

Hou SC, Xiao MB, Ni RZ, et al (2013). Serum GP73 is complementary to AFP and GGT-II for the diagnosis of hepatocellular carcinoma. Oncol Lett, 6, 1152-8.

Kladney RD, Bulla GA, Guo L, et al (2000). GP73, a novel golgi-localized protein upregulated by viral infection. Gene, 249, 53-65.

Lok AS, Sterling RK, Everhart JE, et al (2010). Des-gammacarboxy prothrombin and alpha-fetoprotein as biomarkers for the early detection of hepatocellular carcinoma. Gastroenterology, 138, 493-502.

Liu X, Wan X, Li Z, et al (2011). Golgi protein 73 (GP73), a useful serum marker in liver diseases. Clin Chem Lab Med, 49, 1311-6.

Li X, Zhong X, Chen ZH, et al (2014). Hepatitis B virus DNA negativity acts as a favorable prognostic factor in hepatocellular carcinoma patients. Asian Pac J Cancer Prev, 15, 9635-41.

Marrero JA, Feng Z, Wang Y, et al (2009). Alpha-fetoprotein, des-gamma carboxyprothrombin, and lectin-bound alpha-fetoprotein in early hepatocellular carcinoma. Gastroenterology, 137, 110-8.

Mao Y, Yang H, Xu H, et al (2010). Golgi protein 73 (GOLPH2) is a valuable serum marker for hepatocellular carcinoma. Gut, 59, 1687-93.

Masuzaki R, Karp SJ, Omata M (2012). New serum markers of hepatocellular carcinoma. Semin Oncol, 39, 434-39.

Munaf A, Memon MS, Kumar P, et al (2014). Comparison of viral hepatitis-associated hepatocellular carcinoma due to $\mathrm{HBV}$ and $\mathrm{HCV}$-cohort from liver clinics in Pakistan. Asian Pac J Cancer Prev, 15, 7563-7.

Ozkan H, Erdal H, Tutkak H, et al (2011). Diagnostic and prognostic validity of golgi protein 73 in hepatocellular carcinoma. Digestion, 83, 83-8.

Qiao Y, Chen J, Li X, et al (2014). Serum GP73 is also a biomarker for diagnosing cirrhosis in population with chronic HBV infection. Clin Biochem, 47, 216-22.

Riener MO, Stenner F, Liewen H, et al (2009). Golgi phosphoprotein 2 (GOLPH2) expression in liver tumors and its value as a serum marker in hepatocellular carcinomas. Hepatology, 49, 1602-9.

Stefaniuk P, Cianciara J, Wiercinska-Drapalo A (2010). Present and future possibilities for early diagnosis of hepatocellular carcinoma. World J Gastroenterol, 16, 418-24.

Sherman M (2010). Hepatocellular carcinoma: epidemiology, surveillance, and diagnosis. Semin Liver Dis, 30, 3-16.

Sun Y, Yang H, Mao Y, et al (2011). Increased golgi protein 73 expression in hepatocellular carcinoma tissue correlates with tumor aggression but not survival.J Gastroenterol Hepatol, 26, 1207-12.

Shan SG, Gao YT, Xu YJ, et al (2013). Gradually increased golgi protein 73 expression in the progression of benign liver diseases to precancerous lesions and hepatocellular carcinoma correlates with prognosis of patients. Hepatol Res, 43, 1199-210.

Somboon K, Siramolpiwat S, Vilaichone RK (2014). Epidemiology and survival of hepatocellular carcinoma in the central region of Thailand. Asian Pac J Cancer Prev, 15, 3567-70.

Sangmala P, Chaikledkaew U, Tanwandee T, Pongchareonsuk $P$ (2014). Economic evaluation and budget impact analysis of the surveillance program for hepatocellular carcinoma in Thai chronic hepatitis B patients. Asian Pac J Cancer Prev, 15, 8993-9004.

Thun MJ, DeLancey JO, Center MM, et al (2010). The global burden of cancer: priorities for prevention. Carcinogenesis, 31, 100-10.

Wang M, Long RE, Comunale MA, et al (2009). Novel fucosylated biomarkers for the early detection of hepatocellular carcinoma. Cancer Epidemiol Biomarkers Prev, 18, 1914-21.

Witjes CD, van Aalten SM, Steyerberg EW, et al (2013). Recently introduced biomarkers for screening of hepatocellular carcinoma: a systematic review and meta-analysis. HepaInter, 7, 59-64.

Wang NY, Wang C, Li W, et al (2014). Prognostic value of serum AFP,AFP-L3, and GP73 in monitoring short-term treatment response and recurrence of hepatocellular carcinoma after radiofrequency ablation. Asian Pac J Cancer Prev, 15, 1539-44.

Wang Y, Yang H, Xu H, et al (2014). Golgi protein 73, not Glypican-3, may be a tumor marker complementary to $\alpha$-Fetoprotein for hepatocellular carcinoma diagnosis. $J$ 
Gastroenterol Hepatol, 29, 597-602.

Xu H, Tian YN, Dun BY, et al (2014). A novel monoclonal antibody induces cancer cell apoptosis and enhances the activity of chemotherapeutic drugs. Asian Pac J Cancer Prev, 15, 4423-8.

Yamashita T, Forgues M, Wang W, et al (2008). EpCAM and $\alpha$-fetoprotein expression defines novel prognostic subtypes of hepatocellular carcinoma. Cancer Res, 68, 1451-61.

Yamamoto K, Imamura H, Matsuyama Y, et al (2010). AFP, AFP-L3, DCP, and GP73 as markers for monitoring treatment response and recurrence and as surrogate markers of clinicopathological variables of HCC. J Gastroenterol, 45, 1272-82.

Zhao XY, Li N, Ding HG, Jiang FF (2010). Detection and evaluation of serum GP73, a resident golgi glycoprotein, as a marker in diagnosis of hepatocellular carcinoma. Chin J Oncol, 32, 943-6.

Zhou Y, Yin X, Ying J, Zhang B (2012). Golgi protein 73 versus alpha-fetoprotein as a biomarker for hepatocellular carcinoma: a diagnostic meta-analysis. BMC Cancer, $\mathbf{1 2}, 17$.

Zhang A, Cao B (2012). Generation and characterization of an anti-GP73 monoclonal antibody for immunoblotting and sandwich ELISA. J Biome Res, 26, 467-73. 